\title{
Impact des conditions d'usinage sur la zone du matériau affectée par le procédé
}

\author{
Stéphanie Bissey-Breton ${ }^{1}$, José Farré1,a ${ }^{1,}{\text { Vincent } \text { Vignal }^{2} \text { et Nicolas Mary }}^{2}$ \\ 1 CEA, Centre de Valduc, 21120 Is-sur-Tille, France \\ 2 ICB, UMR 5209 CNRS-Université de Bourgogne, BP 47870, 21078 Dijon Cedex, France
}

Reçu le 15 mars 2007, accepté le 7 juin 2007

\begin{abstract}
Résumé - Les procédés d'usinage peuvent créer des contraintes résiduelles à la surface des pièces usinées et ainsi modifier la microstructure et la texture de la matière proche de la surface. De tels changements sont importants pour l'étude du comportement des pièces au cours du temps. Il est alors nécessaire de quantifier les contraintes résiduelles, les composantes de texture et de déterminer la microstructure induite par l'usinage pour comprendre et prédire le comportement des surfaces considérées. Dans cette étude, le matériau cuivre pur a été choisi et considéré comme un «système modèle ». En tournage, l'influence du rayon de bec, de la vitesse de coupe et de la présence de lubrifiant sur le champ de contraintes et la texture a été quantifiée à l'aide de techniques DRX. Les modifications micro-structurales ont été évaluées à l'aide de la microscopie électronique à balayage. Enfin, le comportement électrochimique de la surface a été étudié en utilisant la technique de la micro-cellule électrochimique.
\end{abstract}

Mots clés : Tournage / zone affectée / contraintes

\begin{abstract}
Impact of cutting conditions on the affected zone of the machined material. Machining processes can introduce residual stresses at the surface of machined workpieces and can modify the microstructure and texture in a volume close to the surface. Such changes are important when controlling the behaviour of these workpieces during the workpiece lifetime. It is thus necessary to quantify residual stresses and texture components and to determine the machining-induced microstructure to understand and predict the behaviour of modified surfaces. In this study, particular attention was paid to pure copper considered as a modelling system. The influence of the nose radius, the cutting speed and the lubricant on the surface stress field and the surface texture was quantified by means of XRD techniques. The microstructural modifications at the specimen surface and in depth were evaluated using scanning electron microscopy. The electrochemical behaviour of modified surfaces were studied from local measurements using the electrochemical microcell technique.
\end{abstract}

Key words: Machining / turning / surface / stress / microstructure / texture / cutting / lubricant / electrochemical behaviour / microcell technique

\section{Introduction}

La précision des formes générées par usinage et la qualité de l'état de surface obtenue dépendent d'une interaction complexe entre les différents éléments supportant d'une part la surface usinée, et d'autre part l'outil de coupe. Les défauts générés en surface sont classés en différentes catégories : les défauts d'ordre 1 à 4 constituent des défauts de type géométrique alors que les défauts d'ordres 5 et 6 peuvent être qualifiés de métallurgiques. Le projet présenté, conduit en collaboration entre le CEA et

\footnotetext{
a Auteur correspondant : jose.farre@cea.fr
}

l'Université de Bourgogne, s'attache à la compréhension des relations paramétrie de coupe/défauts métallurgiques présents en sous-couche de surface (ordres 5 et 6 ).

Plusieurs études ont été menées afin de décrire l'influence des conditions de coupe sur les contraintes résiduelles et l'orientation cristallographique. Sur un cristal unique, les effets de la profondeur de passe et de l'orientation cristallographique sur les propriétés physiques du matériau ont été étudiés à partir des évolutions constatées en termes de densité de dislocations, microdéformations, microdureté ou déformation de la structure [1]. L'influence de l'orientation cristallographique sur les mécanismes de coupe a constitué le sujet de 
Tableau 1. Paramètres d'essais d'usinage des échantillons de cuivre.

\begin{tabular}{cccccc}
\hline Échantillons & Profondeur de passe $(\mathrm{mm})$ & Lubrification & Outils & $\Delta T\left({ }^{\circ} \mathrm{C}\right)$ & Puissance de coupe $(\mathrm{W})$ \\
\hline Cuivre 1 & 0,05 & Oui & Carbex VCGX & 4,5 & 20 \\
Cuivre 2 & 0,3 & Non & Carbex VCGX & 65 & 132 \\
Cuivre 3 & 0,3 & Oui & Masnada & $/$ & 63 \\
\hline
\end{tabular}

nombreuses recherches [2,3]. Dans le cas de polycristaux, plusieurs auteurs ont montré que la géométrie de l'arête de coupe possède un impact direct sur les niveaux de contraintes générés en tournage dur de finition [4] : l'augmentation du rayon d'arête génère des efforts de coupe plus importants. La vitesse d'avance a également un impact sur les contraintes résiduelles en surface [5]. En outre, l'arête de l'outil constitue le facteur principal d'influence du profil de contraintes résiduelles $[6,7]$. Des modèles thermo-élastiques ou thermo-élastoplastiques sont développés sur la base de résultats expérimentaux, pour prédire les profils de contraintes résiduelles existant sur et juste en dessous de la surface nouvellement créée [8]. Ces simulations numériques donnent des indications intéressantes sur la façon de sélectionner les valeurs des paramètres de coupe à mettre en ouvre pour réduire les contraintes résiduelles de tension [9]. D'autre part, les chercheurs ont montré qu'une surface usinée et une sous-couche de très haute qualité peuvent être obtenues en prenant aussi en compte le type de microstructure du matériau [10].

Dans notre cas, une étude exploratoire a permis de caractériser l'influence de paramètres d'usinage sur le matériau, de dégager des réponses significatives telles que des mesures de rugosité, de puissance consommée au cours de la coupe, de contraintes ou de réactivité de la surface, mais également d'extraire trois domaines dans la zone affectée, correspondant à des phénoménologies différentes.

Cet article vise donc à présenter les résultats obtenus lors de cette étude : observation de domaines de phénoménologies différentes, extraction des conditions de coupe impactant la sous-couche sur des épaisseurs micrométriques et premiers résultats quant à l'impact des conditions de coupe sur la réactivité de la surface.

\section{Mise en œuvre expérimentale}

Divers essais de chariotage ont été menés sur du cuivre pur, matériau qui ne présente pas de changement de phase. Certains paramètres de coupe étaient fixés, tels que la vitesse de coupe $\left(V_{\mathrm{c}}=150 \mathrm{~m} \cdot \mathrm{min}^{-1}\right)$ et l'avance $\left(f=0,05 \mathrm{~mm} \cdot \mathrm{tr}^{-1}\right)$. Les valeurs des autres paramètres sont présentées dans le tableau 1. Pendant ces essais, il a été possible de mesurer aussi bien l'élévation de température à une distance d'environ $0,5 \mathrm{~mm}$ de la pointe de l'outil (valeurs de $\Delta T$ déterminées en comparaison avec la température ambiante) et la puissance moyenne consommée au cours de la coupe.

La représentation schématique de la géométrie du processus de coupe de la figure 1 montre l'outil en position d'usinage de type chariotage. Le repère utilisé pour

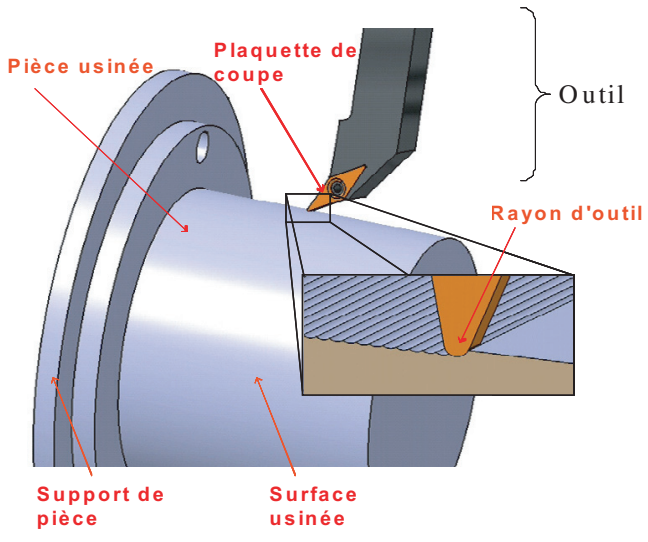

Fig. 1. Configuration de chariotage.

a a) surface usinée

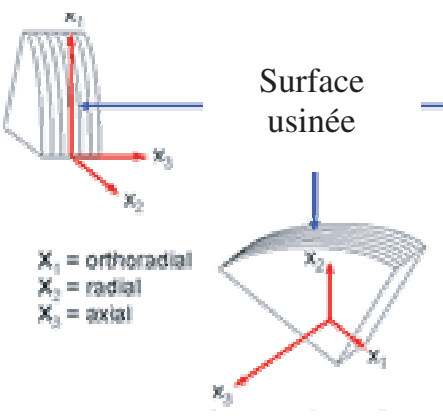

b) surface en coupe

Fig. 2. Repère utilisé et surfaces étudiées.

les analyses est présenté sur la figure 2. Les mesures ou analyses ont été menées sur trois surfaces différentes, notamment la surface usinée qui est perpendiculaire à l'axe radial, et les deux surfaces des sections coupées, respectivement perpendiculaires à l'axe radial et à l'axe orthoradial. Les deux sections en coupe ont été polies à la pâte diamantée $1 \mu \mathrm{m}$ avant de réaliser des mesures ou des observations. Les surfaces ont tout d'abord été analysées grâce à des moyens de profilométrie optique afin de mesurer les états de surface et de microscopie électronique à balayage afin d'évaluer les modifications microstructurales.

D'autre part, la texture de surface a été quantifiée grâce à un goniomètre et un tube Co : goniomètre D8 Discover avec un système de détection de diffraction $2 \mathrm{D}$, et un tube Co $(\lambda \mathfrak{g} 0,1788 \mathrm{~nm})$ fonctionnant sous $40 \mathrm{kV}$ et $30 \mathrm{~mA}$. Le diamètre du faisceau de rayons $\mathrm{X}$ était de $50 \mu \mathrm{m}$. Les mesures ont été réalisées sur une plage angulaire de $-50^{\circ}$ à $+50^{\circ}$ par pas de $5^{\circ}$ et le temps 


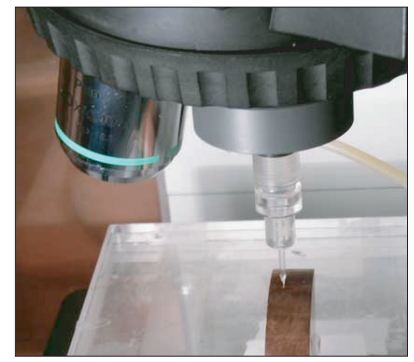

Fig. 3. Micro-capillaire en contact avec la surface usinée.

Tableau 2. Évolution de l'épaisseur de la zone affectée et de l'indice de texture.

\begin{tabular}{ccc}
\hline Échantillons & $\begin{array}{c}\text { Épaisseur de la zone } \\
\text { affectée }(\mu \mathrm{m})\end{array}$ & $\begin{array}{c}\text { Indice de texture } \\
\text { (a.u.) }\end{array}$ \\
\hline Cuivre 1 & $2,5 \pm 1$ & 3,5 \\
Cuivre 2 & $4,6 \pm 2$ & 6,9 \\
Cuivre 3 & $1,5 \pm 1$ & 1,6 \\
\hline
\end{tabular}

d'acquisition de chaque mesure était de 60 s. Les diffractogrammes ont été analysés de la façon suivante : les pics de diffraction ont tout d'abord été atténués grâce à une transformation de Fourier et leur position a été déterminée par la méthode de la corde. Les valeurs de contraintes ont ensuite été déterminées par la méthode des $\sin ^{2} \psi[10]$. L'angle $\psi$ varie dans notre cas entre $-82,5^{\circ}$ et $+82,5^{\circ}$ (120 s par pas $)$.

Enfin, des mesures électrochimiques locales ont été menées grâce à la technique de la micro-cellule électrochimique [11]. Cette technique, qui consiste à limiter la surface de l'échantillon en contact avec l'électrolyte par l'utilisation d'un micro-capillaire en verre, permet la sélection et la caractérisation de sites contenant des phases métalliques, d'hétérogénéités métallurgiques, etc. Le diamètre du micro-capillaire varie de 30 à $100 \mu \mathrm{m}$ (Fig. 3).

La micro-cellule est fixée sur la tourelle d'un microscope pour positionner précisément le micro-capillaire sur la surface. Ce système est ensuite placé dans une cage de Faraday. Un potentiel cathodique $(-300 \mathrm{mV})$ est tout d'abord appliqué pendant deux minutes, puis les courbes de polarisation potentiodynamiques sont établies avec une vitesse de balayage de $1 \mathrm{mV} \cdot \mathrm{s}^{-1}$ dans une solution $1 \mathrm{M}$ $\mathrm{NaClO}_{4}$.

\section{Résultats et discussion}

\subsection{Microstructure de l'échantillon usiné}

L'existence d'un volume affecté en proximité de la surface usinée a été révélée. L'épaisseur de cette zone dépend des conditions de coupe, comme cela apparaît dans le tableau 2. Sur les surfaces usinées, il est également à noter que l'indice de texture croît lorsque l'épaisseur de la zone affectée augmente.

La profondeur de passe la plus importante associée à l'absence de lubrifiant induit l'épaisseur affectée la plus
Tableau 3. Valeurs des contraintes orthoradiales et axiales sur la surface usinée.

\begin{tabular}{ccc}
\hline Échantillons & $\begin{array}{c}\text { Contraintes } \\
(\mathrm{MPa}) \text { orthoradiales }\end{array}$ & $\begin{array}{c}\text { Contraintes axiales } \\
(\mathrm{MPa})\end{array}$ \\
\hline Cuivre 1 & $90 \pm 7$ & $98 \pm 6$ \\
Cuivre 2 & $100 \pm 12$ & $104 \pm 12$ \\
Cuivre 3 & $37 \pm 11$ & $-22 \pm 7$ \\
\hline
\end{tabular}

grande, d'environ 4,6 $\mu \mathrm{m}$ pour l'échantillon Cuivre 2. Des petits grains d'une taille moyenne d'environ $3,5 \mu \mathrm{m}$ ont été observés au sein de la couche formée sur cet échantillon (Figs. 4a,b), montrant que la microstructure en proche surface est significativement différente de celle du matériau à cour. Concernant la surface usinée (Fig. 4c), les limites des grains sont marquées et la taille de grain mesurée est d'environ $8 \mu \mathrm{m}$. De telles modifications n'ont par contre pas été observées sur les deux autres échantillons.

\subsection{Contraintes de surface générées par l'usinage}

Les résultats obtenus sur Cuivre 1 et Cuivre 2 montrent que la lubrification et la profondeur de passe n'ont pratiquement pas d'influence sur les valeurs de contraintes. Des contraintes de tension d'environ $100 \mathrm{MPa}$ ont été calculées dans les trois directions (Tab. 3). Au contraire, le type d'outil utilisé semble avoir un effet sur l'état mécanique du matériau. Les contraintes de surface sont moins importantes en utilisant l'outil Masnada (Tab. 3) et des contraintes de compression ont été obtenues dans la direction axiale. Ces différences peuvent être mises en relation avec la composition du matériau de l'outil et à sa géométrie.

Les gradients de contraintes en proche surface de la surface usinée ont été ensuite estimés en considérant une loi exponentielle. Les valeurs de contraintes calculées en surface ( $z=0$ sur Fig. 5 ) sont du même ordre de grandeur pour les trois combinaisons de conditions de coupe (environ -500 MPa). Des différences peuvent être observées sur la forme des courbes : les contraintes décroissent rapidement lorsque l'outil Masnada est utilisé.

\subsection{Contraintes résiduelles mesurées sur les surfaces en coupe}

Sur ces surfaces en coupe (polies mécaniquement), les contraintes diminuent sur une distance d'environ $250 \mu \mathrm{m}$ (Fig. 6) jusqu'à atteindre des valeurs négatives. En s'éloignant de la surface usinée, les contraintes tendent vers une valeur limite correspondant à celle obtenue sur des échantillons polis. Les plus fortes variations de contraintes ont été observées sur Cuivre 2 alors que les plus faibles concernent Cuivre 1. 

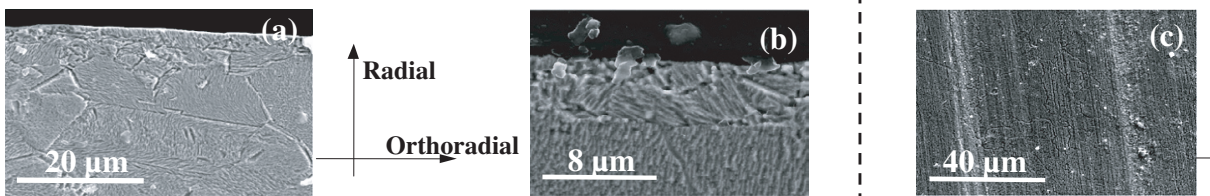

Orthoradial

Axial

Fig. 4. Micrographies de la zone affectée sur Cuivre 2 : (a, b) surface en coupe et (c) surface usinée.
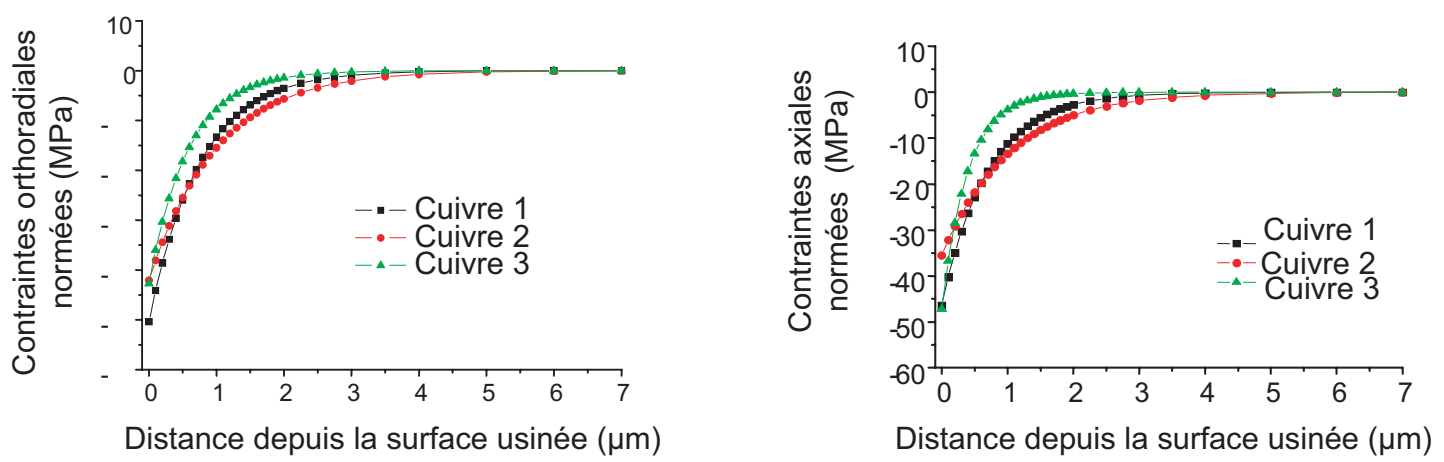

Fig. 5. Évolution des contraintes orthoradiales et axiales de la surface usinée au cœur.
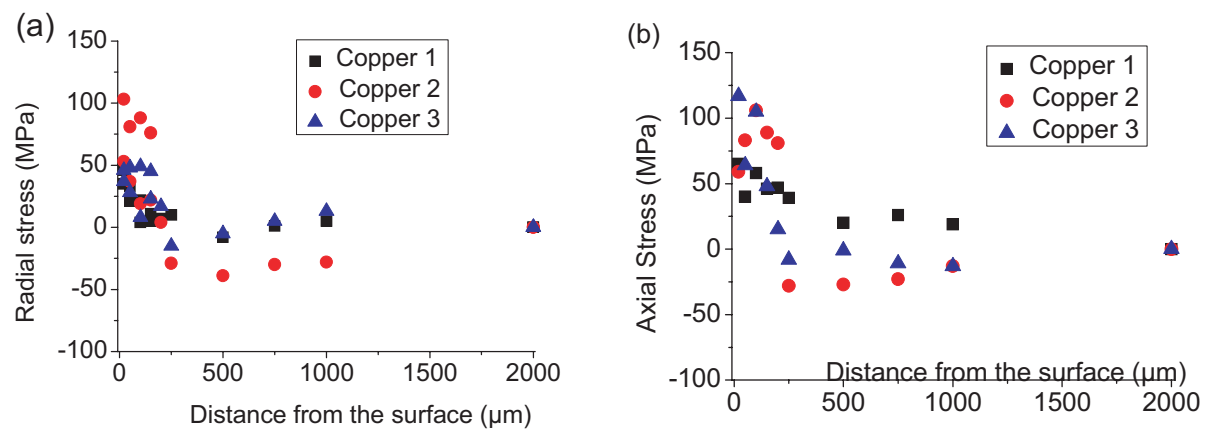

Fig. 6. Évolution des contraintes résiduelles des surfaces en coupe dans les directions (a) radiales et (b) orthoradiales.

\subsection{Comportement électrochimique des surfaces usinées}

Les courbes de polarisation locale ont d'abord été tracées dans $1 \mathrm{M} \mathrm{NaClO}_{4}$ pour les trois échantillons avec un micro-capillaire de diamètre $100 \mu \mathrm{m}$ (Fig. 7). Les courbes obtenues pour Cuivre 2 et Cuivre 3 ont quasiment la même forme. L'utilisation de lubrifiant et de l'outil Masnada peuvent affecter la composition chimique et l'état mécanique de la surface usinée de Cuivre 3 qui présente les plus fortes densités de courant. Par ailleurs, le potentiel de corrosion du Cuivre 1 est décalé d'environ $50 \mathrm{mV} / \mathrm{SCE}$ vers les potentiels anodiques. Cette évolution peut être reliée à l'état mécanique de la surface (le potentiel de corrosion serait plus cathodique lorsque la surface est plus en compression). Les mesures réalisées avec des capillaires plus petits (diamètre : $50 \mu \mathrm{m}$ ) mettent en évidence des différences de comportement dans le domaine anodique entre les creux et les sommets des sillons, mais aucune n'a pu être détectée dans les branches cathodiques.

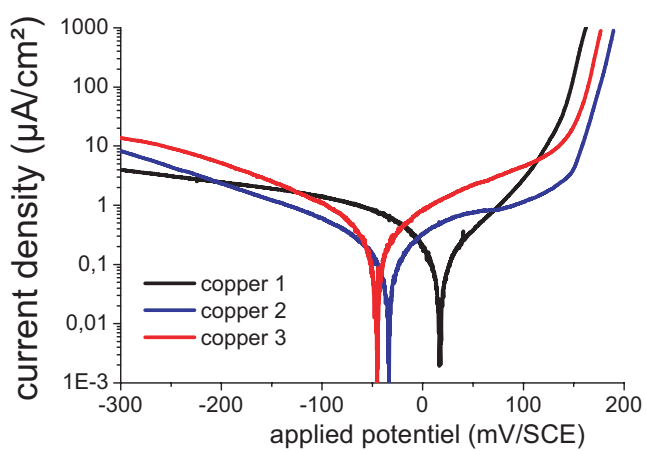

Fig. 7. Courbes de polarisation potentiodynamiques déterminées sur les surfaces usinées dans $1 \mathrm{M} \mathrm{NaCIO}_{4}$ à $1 \mathrm{mV} \cdot \mathrm{s}^{-1}$.

\section{Conclusion}

Ce travail constitue une première étape dans l'étude des relations entre la microstructure et le comportement 
mécanique et chimique de la surface usinée. Les résultats ont pu mener aux conclusions suivantes :

- la microstructure de proche surface est affectée par la profondeur de passe et la lubrification.

- les contraintes résiduelles sont peu influencées par les conditions de coupe. Toutefois, l'évolution du gradient de contraintes près de la surface dépend du type d'outil et de la profondeur de passe.

- les tests électrochimiques montrent que l'échantillon usiné avec la profondeur de passe la plus faible et présence de lubrifiant a le potentiel de corrosion le plus anodique que le changement d'outil réalisé n'affecte pas le comportement à la corrosion de la surface.

\section{Références}

[1] S. To, W.B. Lee, Deformation behaviour of aluminium single crystals in ultraprecision diamond turning, J. Materials Processing Technology 113 (2001) 296-300

[2] K. Ueda, A. Amano, K. Ogawa, H. Takamstu, S. Sakuta, S. Murai, Machining high precision mirrors using newly developed CNC machine, Annals of CIRP 40 (1991) 555565

[3] Z.J. Yuan, J.C. He, X.Y. Yao, The optimum crystal plane of natural diamond tool for precision machining, Annals of CIRP 41 (1992) 65-69
[4] J.D. Thiele, S.N. Melkote, Effect of cutting edge geometry and work-piece hardness on surface generation in the finish hard turning of AISI52100 steel, J. Materials Processing Technology 94 (1999) 216-226

[5] E. Capello, P. Davoli, G. Bassanini, A. Bisi, Residual stresses and surface roughness in turning, J. Engineering Materials Technology 121 (1999) 346-351

[6] M. Jacobson, Surface integrity of hard-turned M50 steel. Proceedings of the Institution of Mechanical Engineers, PartB, J. Engineering Manufacture 216 (2002) 47-54

[7] F. Gunnberg, M. Escursell, M. Jacobson, The influence of cutting parameters on residual stresses and surface topography during hard turning of $18 \mathrm{Mcr} 5$ case carburised steel, J. Materials Processing Technology 147 (2004) $82-90$

[8] K. Jacobus, R.E. DeVor, S.G. Kapoor, MachiningInduced Residual stress: Experimentation and Modelling, J. Manufacturing Science and Engineering 122 (2000) 2031

[9] M. Salio, T. Berruti, G. De Poli, Int. J. Mechanical Sciences 48 (2006) 976-984

[10] H. Dolle, The influence of Multiaxial Stress, Stress Gradient and Elastic Anisotropy on the evaluation of residual stresses by X-rays, J. Applied Crystallography 12 (1979) 489-510

[11] T. Suter, H. Böhni, A new microelectrochemical method to study pit initiation on stainless steels, Electrochimica Acta 42 (1997) 3275-3280 